\title{
Perspectives : The Role of Clinicians in Understanding Secondary Neurulation
}

\author{
Kyu-Chang Wang \\ Neuro-oncology Clinic, Center for Rare Cancers, National Cancer Center, Goyang, Korea
}

During the last two decades, there have been remarkable advances in knowledge regarding secondary neurulation. An increased number of cases of occult spinal dysraphism and progress in basic embryology research have provoked the continuous discovery of new disease entities and the reclassification of occult spinal dysraphic lesions. Examples of such changes are described. The characteristics of secondary neurulation compared with those of primary neurulation are listed and discussed. Our fundamental questions include what the evolutionary significance of secondary neurulation is and what the advantages of having secondary neurulation are. However, our current data and speculations are insufficient to support scientific inference. The direction of future progress of research in this field is predicted. The role of clinicians in this progress is emphasized.

Key Words : Secondary neurulation · Clinician · Embryology · Spinal dysraphism.

\section{INTRODUCTION}

Since the late 19th century, the morphological aspects of secondary neurulation of animals have been described in the literature. Basic researchers have reported the details of morphological events and molecular changes. However, secondary neurulation remained unfamiliar to clinicians, even neuropathologists, until the 1990s. As the number of cases of open neural tube defect (myelomeningocele) decreased and that of closed (occult) neural tube defect (such as lumbosacral lipomatous malformation and 'tethered spinal cord syndrome' in a wider sense, not just thickened filum terminale) increased, attention has gradually shifted from primary neurulation to secondary neurulation. My laboratory research interest also changed from 'surgically induced open neural tube defect' to 'secondary neurulation' in chick embryos in the early 2000s. The characteristics of secondary neurulation compared with those of primary neurulation are listed and discussed. The direction of future progress of research in this field is predicted.

\section{'PRIMARY' VS. 'SECONDARY' NEURAL TUBE FORMATION}

The term 'secondary neurulation' seems to be based on the chronological appearance compared with that of primary neurulation during the development of an embryo. Primary neurulation accompanies dramatic changes in surface struc-

- Received : February 16, 2021 •Accepted : March 2, 2021

- Address for reprints : Kyu-Chang Wang

Neuro-oncology Clinic, Center for Rare Cancers, National Cancer Center, 323 IIsan-ro, Ilsandong-gu, Goyang 10408, Korea

Tel : +82-31-920-0889, Fax : +82-31-920-2799, E-mail : kcwang@snu.ac.kr, ORCID : https://orcid.org/0000-0001-7440-6650

This is an Open Access article distributed under the terms of the Creative Commons Attribution Non-Commercial License (http://creativecommons.org/licenses/by-nc/4.0) which permits unrestricted non-commercial use, distribution, and reproduction in any medium, provided the original work is properly cited. 
tures before secondary neurulation takes place 'silently' under the surface of the embryo, and primary neurulation was well known before secondary neurulation was described. Therefore, it obtained the designation of 'primary'. However, considering the site of the process, the terms 'primary neurulation' and 'secondary neurulation' may not be accepted as granted. The location of secondary neurulation is the caudal cell mass at the area of Hensen's node and primitive streak, which is the center of embryogenesis (the 'primary' or 'primitive' source of embryogenesis) from which basically all the structures of an embryo are derived. It is common that embryologically basic regions give rise to many structures while remaining in a primitive state and later differentiate into other structures. For example, the germinal matrix of the neural tube, especially at the area of ganglionic eminence, remains unchanged until most of the primitive brain structures are made and then changes itself to the ependymal lining and small nests of stem cells. The 'secondary' neural tube originates directly from the center of embryogenesis, Hensen's node and primitive streak, though it is formed later than the primary neural tube. Use of the words 'primary' and 'secondary' may lead to the misconception that the secondary neural tube is a product of the primary neural tube.

\section{CHARACTERISTICS OF SECONDARY NEURULA- TION}

Compared with primary neurulation, secondary neurulation has several unique characteristics.

1) As stated above, secondary neurulation is a later process than primary neurulation. The secondary neural tube starts to form when the caudal neuropore of the primary neural tube begins to close. Therefore, the outcome of neural insults may differ according to the timing.

2) The ventro-dorsal difference in chronological development of the secondary neural tube is less evident than that in the primary neural tube. In the primary neural tube, the development looks more advanced in the ventral part.

3) Secondary neurulation progresses from the node-streak border to the 'caudal direction', whereas primary neurulation is associated with the 'cranial extension' of the notochord from the Hensen's node.

4) There are remarkable surface changes in primary neuru- lation, including folding and fusion. However, the formation of the caudal cell mass and medullary cord does not involve such dramatic surface changes. The mechanism of lumen formation in the neural tube is different from that in the primary neural tube. Hence, it has been believed that secondary neurulation disorders do not involve skin defects. In other words, it has been a common sense that open neural tube defects are not formed at the area of secondary neurulation.

5) Even though the primary and secondary neural tubes are never separate from each other during development, they need 'junctions'. The two structures made by different mechanisms should be functionally connected. The term 'junction' may lead to the misconception that the two separate structures physically unite. Cases of junctional neural tube defect and segmental spinal dysgenesis may show an 'almost separated' appearance between the two neural tubes. This appearance is not a result of poor physical union but a result of a marked reduction in the size of the structure linking the two neural tubes caused by some insults. Normally, the two neighboring neural tubes must be functionally connected and maintain healthy physical continuity.

6) Compared with the neural-inductive role of the notochord in primary neurulation, the neural-inductive role of the notochord in secondary neurulation seems absent. Further investigation will uncover the significance of this discrepancy.

7) There is a strong association between the medullary cord and surrounding mesenchymal tissue (caudal mesenchyme). Changes in caudal mesenchymal activity may disturb the formation of surrounding structures such as the urogenital tract, distal bowel, and abdominal wall. In other words, even though these structures were not directly derived from the caudal cell mass, their anomalies can be caused by anomalous development of the caudal cell mass.

8) There is a regressive phase in secondary neurulation that is absent in primary neurulation. This phase brings about peculiar anomalies (failed regression spectrum of the medullary cord) in secondary neurulation.

Details of each pathogenetic mechanism and its related disease entity are available in other chapters of Pediatric Issues 2020 and 2021. 


\section{EVOLUTIONARY SIGNIFICANCE OF SECONDARY NEURULATION}

I have often had to answer the following questions from students or audiences. What is the evolutionary meaning of secondary neurulation? Why did nature invent secondary neurulation? What are the advantages of having secondary neurulation?

The evolutionary significance and advantages of secondary neurulation are unknown. There are so many questions that we cannot answer. We do not know why the characteristics of progenitor cells in parts of the central nervous system are not the same, as discussed in the chapter 'Embryonal neuromesodermal cells for caudal central nervous system and tissue development'. We do not know how cell fates are determined according to the small compartments in a limited area of a certain structure, as described in the chapter 'Secondary neurulation : cell fate'. Questions about evolutionary significance are more difficult to answer.

We know that the secondary neural tube takes part in more basic and involuntary autonomic functions, such as excretion and sexual functions. Many animals express emotional responses of joy, excitement, fear, vigilance, obedience or aggression with tail movements. In some animals, as written in the chapter 'A tale of the tail', the tails and the secondary neural tube structures play roles in autonomic motor activities (such as grasping branches while moving across trees or maintaining body balance during fast movement). The majority of functions performed by the secondary neural tube and its related structures are basically involuntary but are partially voluntarily controlled, like respiration. There may be some advantages of having a separate unit for those rather autonomic functions. Nevertheless, our current data and speculations are insufficient to support scientific inference.

\section{FUTURE DIRECTION}

As most fields of medical science continue to progress, molecular control mechanisms must be one of the main topics in future research on secondary neurulation. Laboratory experiments on its molecular manipulation will uncover pathogenetic clues for many disease entities.

However, clinicians also have an essential role. Bridging the results of basic research and the clinical findings of associated anomalies will provide solid knowledge in this field. Accordingly, the classification of caudal spinal malformative lesions will be continuously revised. Clinicians may suggest the pathoembryogenetic mechanisms of such lesions, taking preexisting knowledge and newly noted clinical findings into consideration with sound reasoning. These suggestions may be conjectural, controversial and contradictory to previous speculations. Nevertheless, these efforts will promote advances in our understanding of malformative lesions in the caudal spinal area and improvements in the management of patients. For example, terminal myelocystocele was defined as a clinical entity in the middle of the $1980 \mathrm{~s}^{3}$. During the following period of more than two decades, knowledge on its clinical manifestation and strategy for management were refined, and pathoembryogenetic mechanisms were suggested based on its relationship to similar lesions (such as one without "trumpetlike" flaring, terminal myelocele) or similar mechanisms (such as retained medullary cord $)^{1,2,4)}$. These advances are based on the establishment of the clinical entity "terminal myelocystocele" by clinicians. Another example is the open neural tube defect in the area of secondary neural tube. As mentioned above, it has been a common sense that open neural tube defects do not occur at the area of secondary neural tube because secondary neurulation is a process occurring inside of the body (not involving remarkable surface changes which are seen in the primary neurulation). However, as briefly described in the chapter 'Overview of secondary neurulation' and clinically illustrated in more detail in the chapter 'Disorders of secondary neurulation', this concept seems to be changing. Further clinical data collection and reasoning with embryological background may solve the question.

As the concept of secondary neurulation becomes familiar to clinicians, pathoembryogenetic theories regarding caudal spinal malformative lesions are increasing. Our team also found that almost every malformative lesion that has been thought to occur only in the area of the primary neural tube may be present at the area of the secondary neural tube, such as myelomeningocele and limited dorsal myeloschisis.

There are still many unanswered questions. We do not know the exact reason why remote lesions such as esophageal or limb anomalies occur in caudal agenesis or caudal duplication syndrome. We do not understand why spastic bladder is frequently associated with tethered spinal cord, whereas ab- 
normalities in electromyography (signs of lower motor neuron lesion) were found in muscles of the ankle. Although the Currarino triad is included in the scope of caudal agenesis, the role of molecular changes that were shown in the Currarino triad is unknown in the pathoembryogenesis of caudal agenesis.

\section{CONCLUSIONS}

During the last two decades, there have been remarkable advances in knowledge regarding secondary neurulation. An increased number of cases of occult spinal dysraphism and progress in basic embryology research have provoked the continuous discovery of new disease entities and the reclassification of occult spinal dysraphic lesions. Reclassification has accompanied changes in previously held concepts. We have found that almost every lesion in the area of the primary neural tube may occur at the area of secondary neurulation. Limited dorsal myeloschisis and myelomeningocele are examples. Some of the previously described separate entities seem to be grouped in a spectrum of errors occurring at a certain step of embryogenesis. For example, terminal myelocystocele was found to be among the spectrum of consequences of failed regression of the medullary cord. Investigators will continue the job of putting together the pieces of the puzzle of 'disorders of secondary neurulation and related processes'.

We expect that more accelerated advances will be made in the near future. A detailed depiction of the whole process of caudal body formation (including secondary neurulation) and reclassification of disorders of secondary neurulation based on the molecular changes will be achieved. For these changes to occur, interaction between basic researchers and clinicians is essential.

\section{CONFLICTS OF INTEREST}

No potential conflict of interest relevant to this article was reported.

\section{INFORMED CONSENT}

This type of study does not require informed consent.

\section{AUTHOR CONTRIBUTIONS}

\author{
Conceptualization : KCW \\ Writing - original draft : KCW
}

\section{ORCID}

Kyu-Chang Wang https://orcid.org/0000-0001-7440-6650

\section{- Acknowledgements}

I express my sincere gratitude to Dr. Ji Yeoun Lee for her critical review of the manuscript and to Dr. Jeyul Yang for his assistance during the preparation of the manuscript.

\section{References}

1. Kim KH, Lee JY, Wang KC : Secondary neurulation defects-1 : retained medullary cord. J Korean Neurosurg Soc 63 : 314-320, 2020

2. Lee JY, Kim SP, Kim SW, Park SH, Choi JW, Phi JH, et al. : Pathoembryogenesis of terminal myelocystocele: terminal balloon in secondary neurulation of the chick embryo. Childs Nerv Syst 29 : 1683-1688, 2013

3. McLone DG, Naidich TP : Spinal dysraphism: experimental and clinical in Holtzman RN, Stein BM (eds) : The tethered spinal cord. New York: Thieme, 1985, pp14-28

4. Pang D, Zovickian J, Lee JY, Moes GS, Wang KC : Terminal myelocystocele: surgical observations and theory of embryogenesis. Neurosurgery 70 : 1383-1404; discussion 1404-1405, 2012 